\title{
An optimal control model of dendritic cell treatment of a growing tumour
}

\author{
Trisilowati ${ }^{1} \quad$ Scott W. McCue ${ }^{2} \quad$ Dann G. Mallet ${ }^{3}$
}

(Received 17 January 2013; revised 10 June 2013)

\begin{abstract}
A new optimal control model of the interactions between a growing tumour and the host immune system, along with an immunotherapy treatment strategy, is presented. The model is based on an ordinary differential equation model of interactions between the growing tumour and the natural killer, cytotoxic T lymphocyte and dendritic cells of the host immune system, extended through the addition of a control function representing the application of a dendritic cell treatment to the system. The numerical solution of this model, obtained from a multi species Runge-Kutta forward-backward sweep scheme, is described. We investigate the effects of varying the maximum allowed amount of dendritic cell vaccine administered to the system and find that control of the tumour cell population is best effected via a high initial vaccine
\end{abstract}

http://journal.austms.org.au/ojs/index.php/ANZIAMJ/article/view/6654 gives this article, (c) Austral. Mathematical Soc. 2013. Published October 25, 2013, as part of the Proceedings of the 16th Biennial Computational Techniques and Applications Conference. ISSN 1446-8735. (Print two pages per sheet of paper.) Copies of this article must not be made otherwise available on the internet; instead link directly to this URL for this article. 
level, followed by reduced treatment and finally cessation of treatment. We also found that increasing the strength of the dendritic cell vaccine causes an increase in the number of natural killer cells and lymphocytes, which in turn reduces the growth of the tumour.

\section{Contents}

1 Introduction and background

C665

2 Mathematical model

C666

3 Computational implementation

C669

4 Results

C671

5 Discussion

C673

References

C677

\section{Introduction and background}

We study an optimal control treatment strategy for the interactions between a growing tumour and the host immune system. The numerical solution of the model is obtained from a Runge-Kutta forward-backward sweep scheme. An optimal control model is developed by building on an ordinary differential equation (ODE) model of tumour-immune system interactions through the addition of a control function, $\mathfrak{u}$, representing the application of a dendritic cell treatment.

Extensive mathematical research was undertaken into the growth of tumour cell colonies. Of particular interest here is the body of research related to the modelling of tumour interactions with the immune system and treatment 
using immunotherapy $[1,2,3,4,5,6,7,9,10,11,12,13,17$, e.g.]. Kirschner and Panetta [13] illustrated the effect of adoptive cellular immunotherapy through a mathematical model which consists of tumour cells, immune-effector cells and interleukin-2. This model describes under what conditions the tumour is destroyed as a result of the therapy. De Pillis et al. [6] developed a mathematical model describing the growing tumour with combination immune, vaccine and chemotherapy treatments. In addition, Cappucio et al. [3] introduced a mathematical model of tumour immune system interactions focusing on natural killer (NK) and T cell immunity, combined with interleukin21 as an immunotherapy. However, there are very few models which study the effect of dendritic cell (DC) vaccines on a growing tumour [5, e.g.] and these treat the immune system in a different manner to the presented research.

Optimal control theory is a commonly employed strategy to determine a treatment method that guarantees a minimum of some cost (such as monetary costs or harm to the patient) while minimising the tumour cell population [2, $9,10,11,19,21$, e.g.], [20, for a review]. Chemotherapy is commonly studied as the tumour treatment, while immune cell treatment [2, 11, e.g.] is more rarely investigated.

Of particular relevance here is the work of Castiglione and Piccoli [5], who constructed a mathematical model to describe the interaction between immune system cells and tumour cells. They then applied optimal control methods to find the optimal quantity of dendritic cells to be administered to the tumour site. In the current research, we also focus on DC based immunotherapeutic treatment; however, our work is different from Castiglione and Piccoli's since we include NK cells and also describe in more detail the role of dendritic cells in tumour control.

In the following section we present an ODE model of tumour cell interactions with specific components of the immune system, that incorporates a time varying dendritic cell based treatment strategy and also an objective functional that we seek to minimise. We then discuss the numerical scheme itself, a multispecies forward-backward sweep method using a Runge-Kutta ODE 
solver and present a number of important numerical solutions of the optimal control model. Finally, we discuss the results of the model in the context of the tumour growth problem.

\section{Mathematical model}

To describe the interactions between the growing population of tumour cells and specific components of the immune system, we introduce four cell species, namely the number of tumour cells $\mathrm{T}(\mathrm{t})$, NK cells $\mathrm{N}(\mathrm{t})$, dendritic cells $\mathrm{D}(\mathrm{t})$, and $\mathrm{CD} 8^{+} \mathrm{T}$ cells (cluster of differentiation eight) $\mathrm{L}(\mathrm{t})$, where here $t$ is time.

We assume that the growth of the tumour cell population is logistic, while tumour cells are removed due to interactions with $\mathrm{NK}$, dendritic and $\mathrm{CD} 8^{+}$ T cells [15]. NK cells and DCs are normally present in the body, even when no tumour cells are present. After some number of interactions with tumour cells, NK cells and $\mathrm{CD}^{+} \mathrm{T}$ cells become inactive and are removed from consideration. DCs are able to prime the activity of NK cells and $\mathrm{CD}^{+} \mathrm{T}$ cells [18]. Finally, mature $\mathrm{CD} 8^{+} \mathrm{T}$ cells can clear out dendritic cells [5, 22]. Combining these assumptions, we model the tumour-immune system interactions using the system of ODEs

$$
\begin{aligned}
\frac{d T}{d t} & =a T(1-b T)-\left(c_{1} N+j D+k L\right) T, \\
\frac{d N}{d t} & =s_{1}-c_{2} N T+d_{11} \frac{D N}{m_{1}+D}-e N, \\
\frac{d D}{d t} & =s_{2}+l \frac{D T}{m_{2}+T}-f_{1} L D-d_{2} D N-g D, \\
\frac{d L}{d t} & =f_{22} \frac{D T}{m_{3}+T}-h L T-i L,
\end{aligned}
$$

where the parameters are described, along with estimated values and literature sources, in Table 1. 
Table 1: Parameters, values and sources, used in the solution of the model given by equations (1)-(4). Rates $\mathrm{a}, \mathrm{d}_{11}, \boldsymbol{e}, \mathrm{g}$ and $\boldsymbol{i}$ are measured per day, rates $c_{1}, c_{2}, d_{2} f_{1}, f_{22}, h, j, k$ and $l$ are measured per cell per day, $b$ is measured per cell, $s_{1}$ and $s_{2}$ measure cells per day, and $m_{1}, m_{2}$ and $m_{3}$ measure numbers of cells.

\begin{tabular}{llcc} 
Param. & Description & Value & Source \\
\hline $\mathrm{a}$ & Tumour growth rate & $4.31 \times 10^{-1}$ & {$[6]$} \\
$\mathrm{b}$ & Inverse tumour carrying capacity & $2.17 \times 10^{-8}$ & {$[6]$} \\
$\mathrm{c}_{1}$ & NK cell tumour cell kill rate & $3.5 \times 10^{-6}$ & {$[8]$} \\
$\mathrm{c}_{2}$ & NK cell inactivation rate by tumour cells & $1.0 \times 10^{-7}$ & {$[6]$} \\
$\mathrm{d}_{11}$ & Maximum rate of NK cell activation & $5.0 \times 10^{-2}$ & Est. \\
$\mathrm{d}_{2}$ & NK cell dendritic cell kill rate & $4.0 \times 10^{-6}$ & Est. \\
$e$ & Death rate of NK cell & $4.12 \times 10^{-2}$ & {$[14]$} \\
$\mathrm{f}_{1}$ & CD8 $8^{+} \mathrm{T}$ cell dendritic cell kill rate & $1.0 \times 10^{-8}$ & {$[5]$} \\
$\mathrm{f}_{22}$ & Maximum rate of CD8 $8^{+}$T cell activation & $1.0 \times 10^{-2}$ & Est. \\
$g$ & Death rate of dendritic cell & $2.4 \times 10^{-2}$ & {$[22]$} \\
$\mathrm{h}$ & CD8 $8^{+}$T inactivation rate by tumour cells & $3.4 \times 10^{-10}$ & {$[6]$} \\
$\mathrm{i}$ & Death rate of CD8 $8^{+}$T cells & $2.0 \times 10^{-2}$ & {$[6]$} \\
$j$ & Dendritic cell tumour cell kill rate & $1.0 \times 10^{-2}$ & Est. \\
$\mathrm{k}$ & CD8 $8^{+} \mathrm{T}$ cell tumour cell kill rate & $1.0 \times 10^{-7}$ & Est. \\
$\mathrm{s}_{1}$ & Source of NK cells & $1.3 \times 10^{4}$ & {$[14]$} \\
$\mathrm{s}_{2}$ & Source of dendritic cells & $4.8 \times 10^{2}$ & {$[22]$} \\
$l$ & Max. DC recruitment by tumour cells & $1.0 \times 10^{-2}$ & Est. \\
$\mathrm{m}_{1}$ & NK cell activation rate steepness coeff. & $1.0 \times 10^{4}$ & Est. \\
$\mathrm{m}_{2}$ & Tumour cell recruitment steepness coeff. & $1.0 \times 10^{4}$ & Est. \\
$\mathrm{m}_{3}$ & CD8 ${ }^{+}$T cell activation steepness coeff. & $3.0 \times 10^{3}$ & Est. \\
& & &
\end{tabular}


The function $u(t) \geqslant 0$ represents the dendritic cell vaccine treatment rate. Positive values of $u(t)$ correspond with an influx of DCs supplied from outside the system. Hence, equation (3) becomes

$$
\frac{d D}{d t}=s_{2}+l \frac{D T}{m_{2}+T}-f_{1} L D-d_{2} D N-g D+u(t) .
$$

We now introduce an objective functional as part of the optimal control problem. We aim to minimise the tumour burden over the length of time modelled, and also the 'cost' of the treatment, be it harm to the patient, cost to administer treatment, or some other interpretation-here we adopt the $\mathfrak{u}^{2}$ form employed in similar works [6]. Hence we aim to minimise $J[t, u(t)]$, where

$$
J[t, u(t)]=\int_{0}^{t_{f}}\left[T(t)+\frac{B}{2} u^{2}(t)\right] d t
$$

where $t_{f}$ is the specified final time and $B$ is a weighting factor that represents the relative importance of the cost as opposed to the tumour burden, in the minimisation problem. In the simulations we used $B=10^{-3}$, reflecting the relatively higher importance placed on reducing the tumour burden than on the cost of the treatment.

\section{Computational implementation}

To proceed with the solution of the optimal control problem, we first rewrite our system as follows. We seek to solve

$$
\mathbf{x}^{\prime}(\mathrm{t})=\mathbf{g}(\mathrm{t}, \mathbf{x}, \mathrm{u}),
$$

where $\mathbf{x}(t)=[T(t), N(t), D(t), L(t)]^{\top}$ is the vector of solutions of the state equations (1), (2), (5) and (4), and $\mathbf{g}$ is the vector of right hand sides of the 
state equations. Also, we rewrite equation (6) as

$$
J[t, x(t), u(t)]=\int_{0}^{t_{f}} f(t, x, u) d t
$$

where $\mathbf{f}$ is the integrand of equation (6).

Next, we form the Hamiltonian function

$$
H(t, x, u, \lambda)=f(t, x, u)+\lambda^{\top} g(t, x, u) .
$$

where $\boldsymbol{\lambda}$ is the vector of adjoint functions. Then we have the optimality condition

$$
\frac{\partial \mathrm{H}}{\partial \mathrm{u}}=0 \quad \text { at } \quad \mathrm{u}^{*} \Rightarrow \mathrm{f}_{\mathrm{u}}+\lambda^{\top} \mathrm{g}_{\mathrm{u}}=0
$$

as well as the adjoint equation and transversality condition

$$
\lambda^{\prime}=-\frac{\partial H}{\partial x}=-\left(f_{x}+\lambda^{\top} g_{x}\right), \quad \lambda\left(t_{f}\right)=0
$$

We now present the algorithm (the forward-backward sweep method) used to solve the bounded optimal control problem of Section 2. We outline an adapted version of the single equation scheme presented by Lenhart and Workman [16] that allows us to solve the multispecies optimal control problem. Consider the optimal control problem

$$
\begin{gathered}
\min _{\mathfrak{u}} \int_{t_{0}}^{t_{f}} f[t, x(t), u(t)] d t, \\
\text { subject to } \quad x^{\prime}=g(t, x(t), u(t)), \quad x\left(t_{0}\right)=x_{0},
\end{gathered}
$$

where $\mathbf{x} \in \mathbb{R}^{n \times 1}, u \in \mathbb{R}$, and $n$ is the number of state equations. Let $\mathrm{X} \in \mathbb{R}^{\mathrm{n} \times(\mathrm{N}+1)}, \Lambda \in \mathbb{R}^{\mathrm{n} \times(\mathrm{N}+1)}, \mathrm{U} \in \mathbb{R}^{\mathrm{N}+1}$, where $\mathrm{N}+1$ is the number of time steps to be taken, $\mathbf{X}$ is the approximation to $\mathbf{x}$ at the $\mathbf{N}+1$ time steps, and $\boldsymbol{\Lambda}$ is a matrix of the adjoint equations. Then, to solve the optimal control problem: 
1. Discretise the time domain into $\mathbf{N}+1$ equidistant mesh points $t_{1}$, $t_{2}, \ldots, t_{N+1}$

2. Make an initial guess $\mathbf{U}$, for $\boldsymbol{u}$ over the interval;

3. Using the initial condition $\mathbf{X}_{i, 1}=\mathbf{x}_{0}, \boldsymbol{i}=1, \ldots, \mathbf{N}+1$, and $\mathbf{U}$, solve equation (7) forward in time using the order four Runge-Kutta method to obtain the next approximation for $\mathbf{X}$;

4. Using the transversality condition $\boldsymbol{\Lambda}_{i, \mathrm{~N}+1}=\lambda\left(\mathrm{t}_{\mathrm{f}}\right)=0$ and the values for $\mathbf{U}$ and $\mathbf{X}$, solve equation (9) backward in time to obtain the next approximation for $\boldsymbol{\Lambda}$;

5. Update $\mathbf{U}$. If values of $\mathbf{U}$ at this iteration and the last are not sufficiently close, then return to step 3 using the updated $\mathbf{X}$ and $\boldsymbol{\Lambda}$ values. If values are sufficiently close, then the control is optimal: output $\mathbf{X}$ and $\mathbf{U}$ as approximations to $\mathbf{x}$ and $\mathbf{u}$.

\section{Results}

In this section, the optimal control problem set up in Section 2 is solved using the forward-backward sweep method as discussed in Section 3.

In Figure 1 we show a plot of the tumour cell population using a large initial value of tumour cells, demonstrating the impact of the DC vaccine based control strategy. The dashed line shows the tumour population growth resulting from no control, while the solid line (more easily seen inset) shows the tumour cell population eradication resulting from the optimal control vaccine strategy. With the initial value $T_{0}=5 \times 10^{4}$ cells, without vaccine, tumour cells grow to the nonzero tumour equilibrium after around 120 days. However, the tumour is eliminated in just under 40 days using the optimal control strategy. The inset plot shows that the tumour cell population is completely removed within 40 days whereas without control, the number of tumour cells remains high at this time. The dendritic cell vaccine to be 


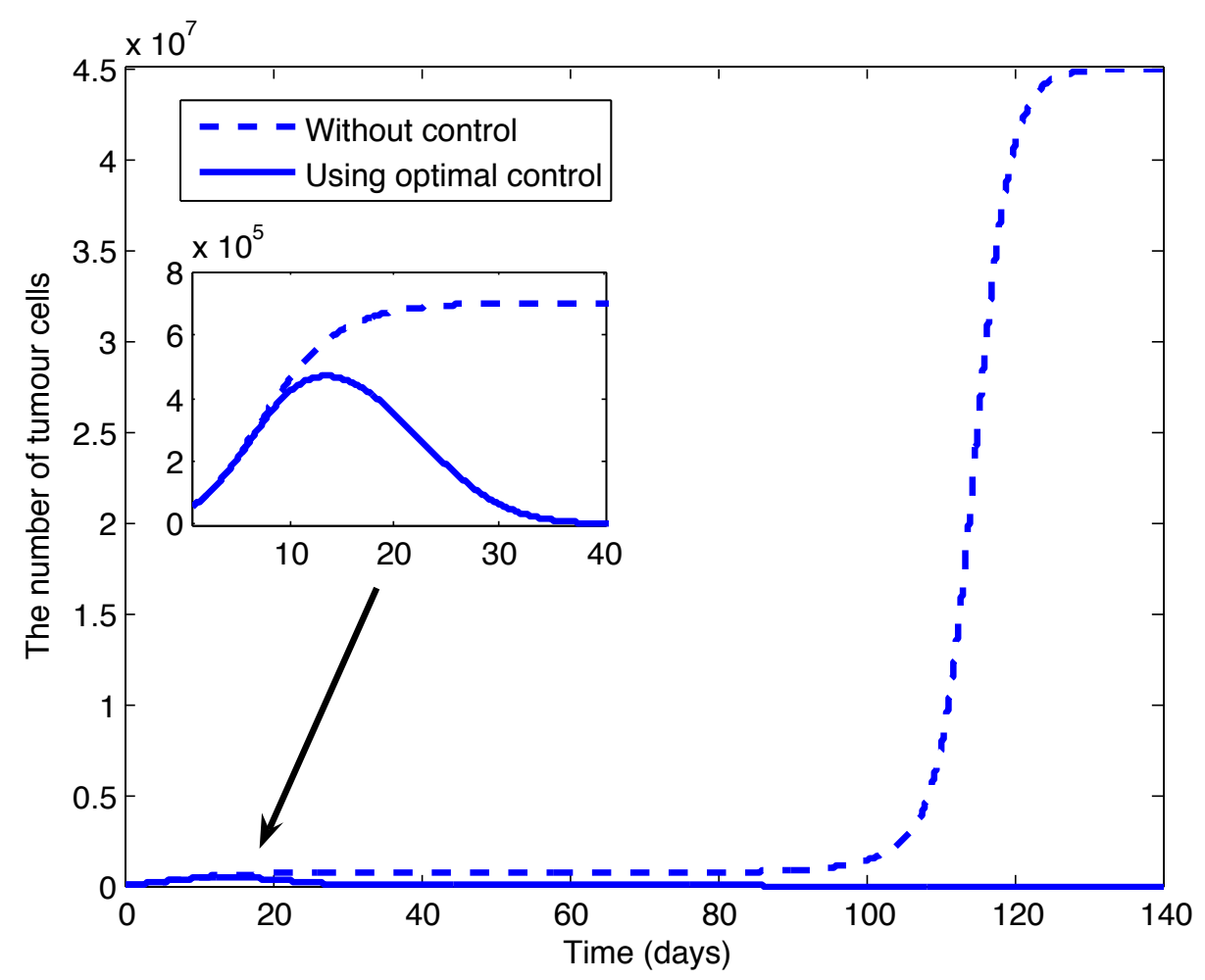

Figure 1: Tumour cell population evolution using a large initial tumour cell population, showing the growth with no DC treatment (dashed) and the impact of the DC based vaccine (solid). 


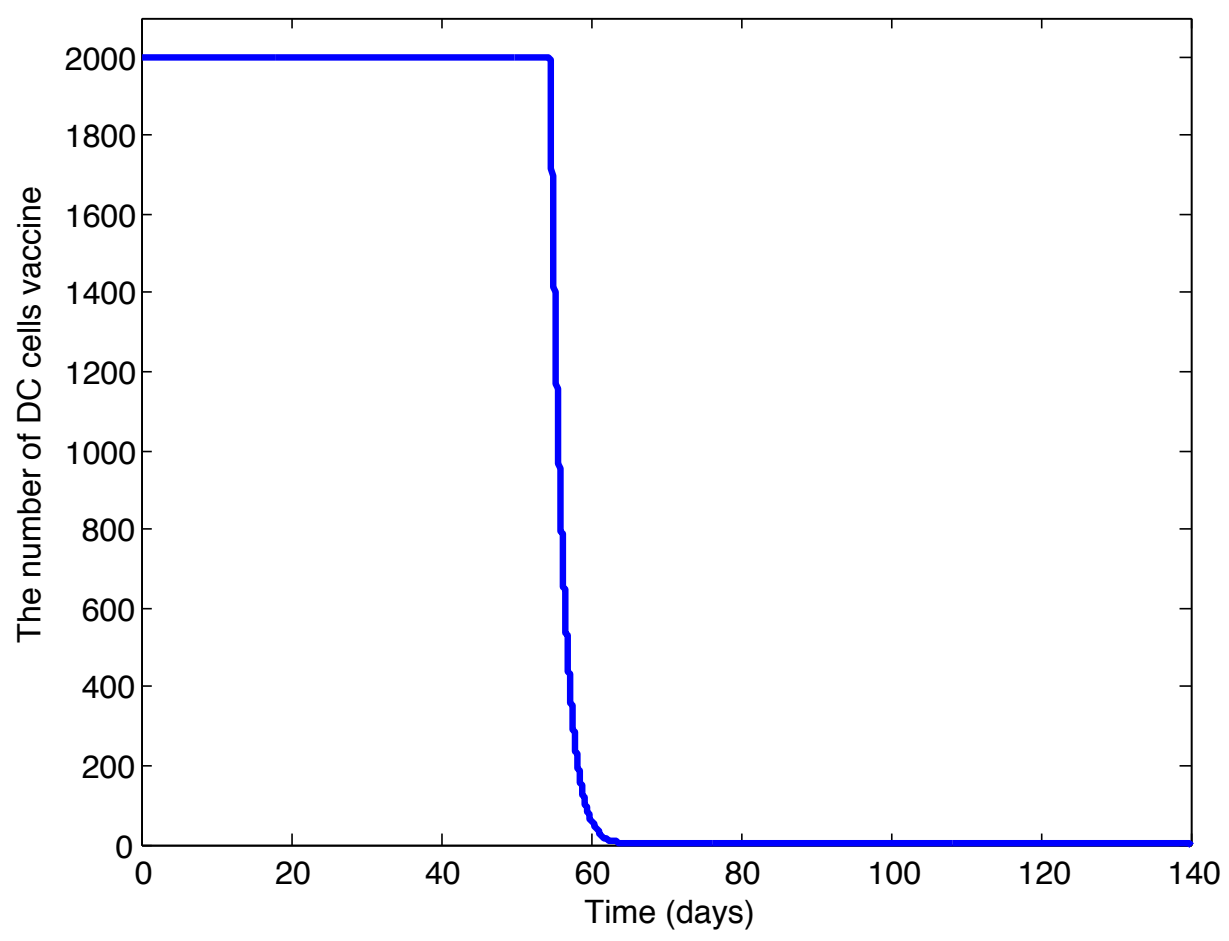

Figure 2: The optimal control, $\mathfrak{u}$, used for solutions in Figure 1.

administered during this treatment is seen in Figure 2. To eliminate the tumour cells as shown in Figure 1, the maximum vaccine treatment should be administered for approximately the first 58 days, after which the treatment should reduce sharply and finally stop at day 62 .

Increasing the strength of the DC vaccine not only impacts the tumour cell population, it also causes increases in the numbers of NK cells (see Figure 3) and $\mathrm{CD}^{+} \mathrm{T}$ cells (see Figure 4). These effects in turn reduce the tumour cell population as shown in Figure 5. The effect is very significant in increasing the number of $\mathrm{CD} 8^{+} \mathrm{T}$ cells, consequently reducing the tumour burden. This particular numerical solution shows that DCs alter the population of $\mathrm{CD}^{+}$ 


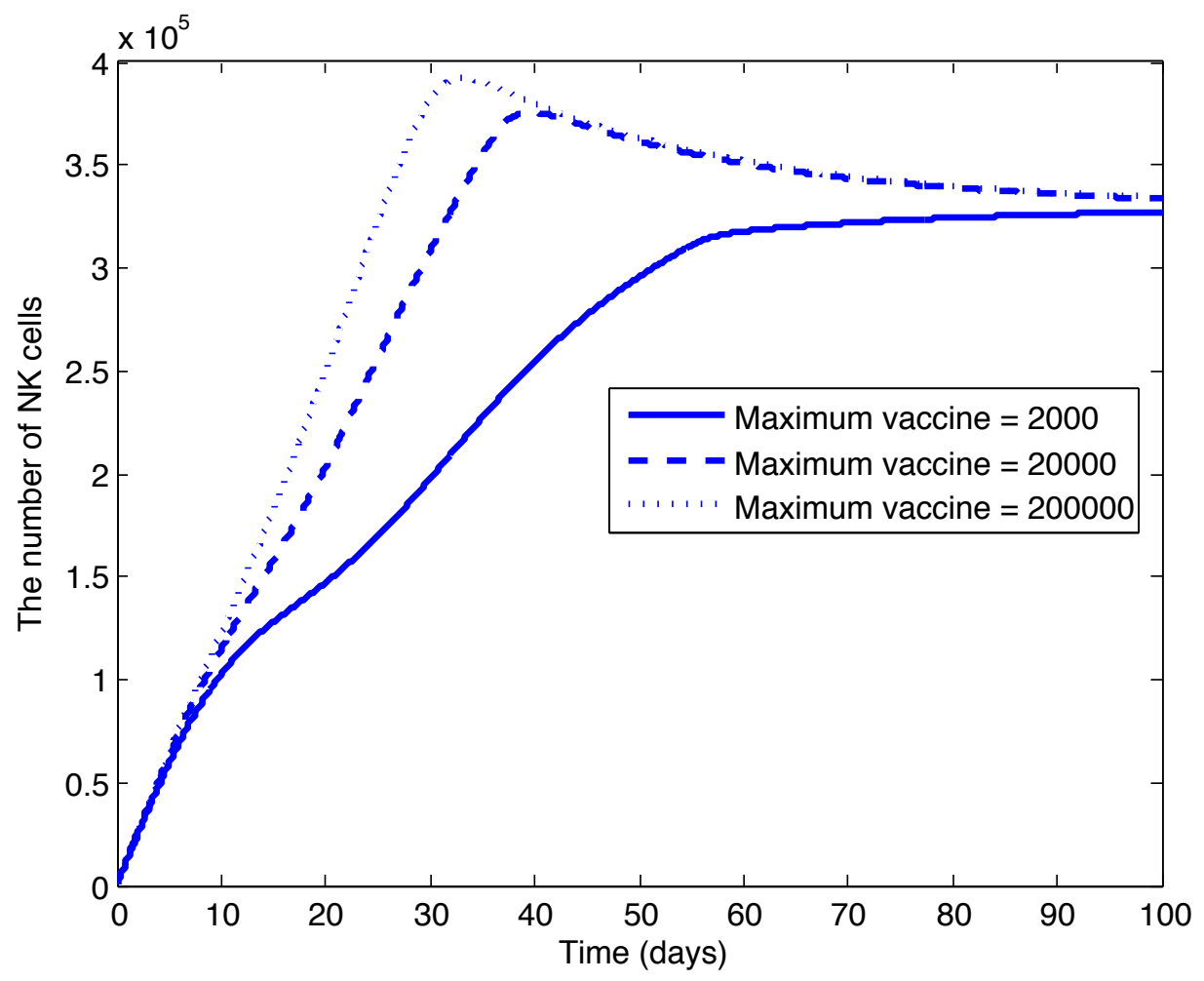

Figure 3: Impact of the DC vaccine on NK cell evolution for maximum vaccine levels $u_{\max }=2$ (solid), 20 (dashed) and 200 (dotted). Increasing $u_{\max }$ increases the peak NK level and decreases the time to reach that peak. 


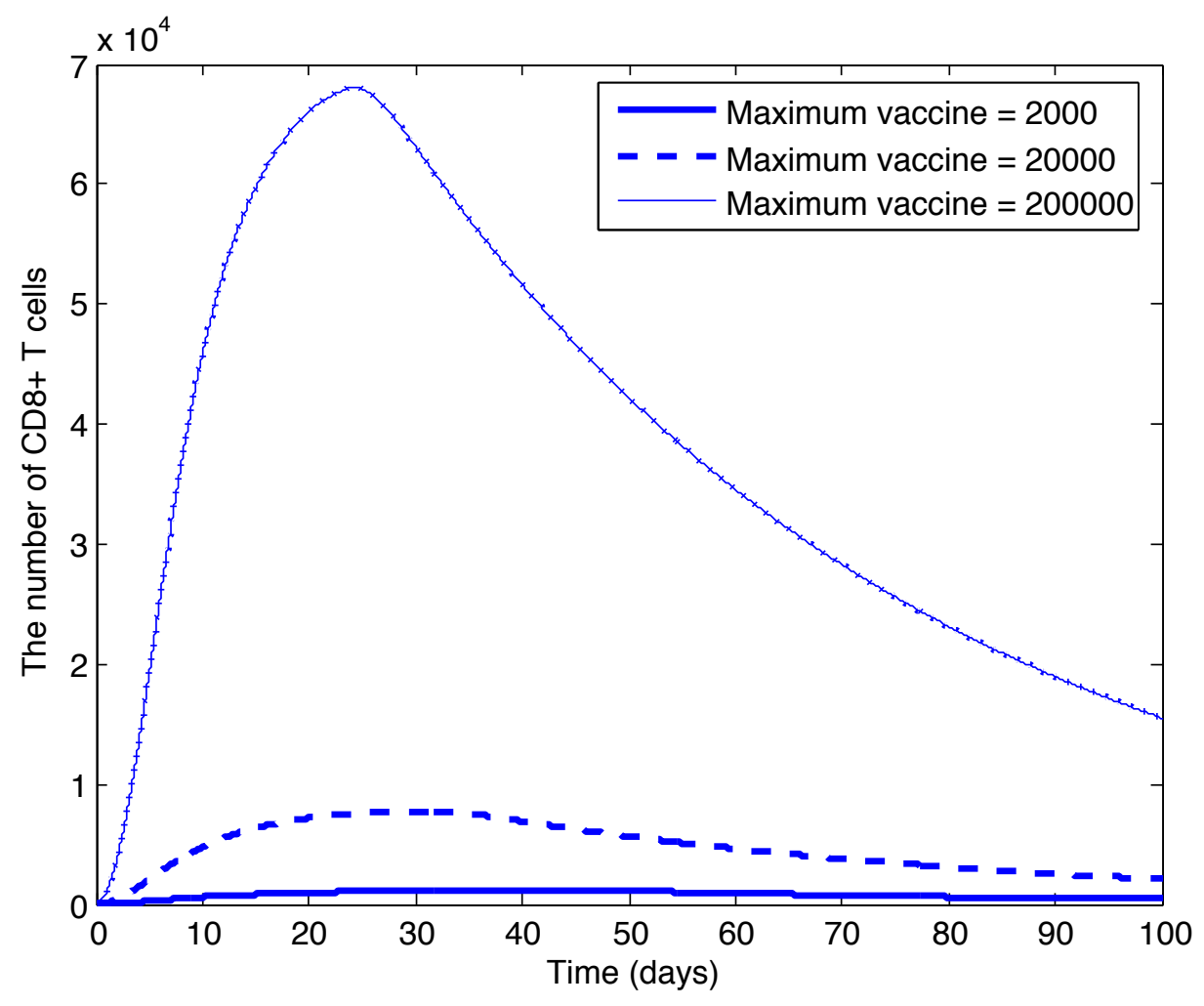

Figure 4: Impact of the $\mathrm{DC}$ vaccine on $\mathrm{CD} 8^{+} \mathrm{T}$ cell evolution for maximum vaccine levels $u_{\max }=2$ (solid), 20 (dashed) and 200 (dotted). Increasing $u_{\max }$ increases the peak $\mathrm{CD} 8^{+} \mathrm{T}$ cell level and decreases the time to reach that peak. 


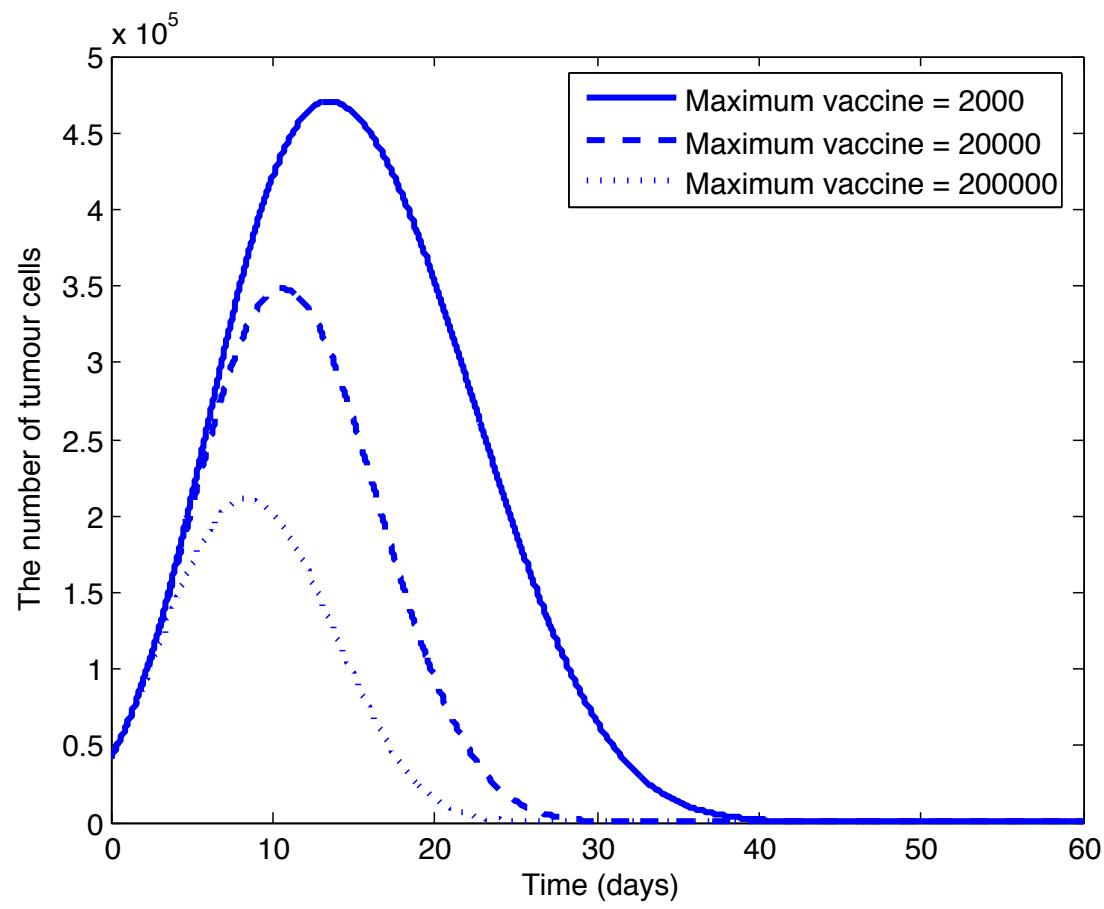

Figure 5: Impact of the DC vaccine on the tumour cell evolution for maximum vaccine levels $u_{\max }=2$ (solid), 20 (dashed) and 200 (dotted). Increasing $u_{\max }$ decreases the peak tumour cell level and the time to reach that peak.

T cells much more than NK cells.

\section{Discussion}

We have presented a new optimal control model of dendritic cell treatment of a growing population of tumour cells. The model builds on the previous modelling literature in terms of the type of model and the optimal control approach, but differs from existing work through the explicit treatment of DCs 
in addition to NK and $\mathrm{CD} 8^{+} \mathrm{T}$ cells. The model was solved using a multispecies implementation of the forward-backward sweep method for solving optimally controlled systems of ODEs.

Our results focus on investigating the effects of varying the maximum allowed amount of DC vaccine administered to the system. We found that the best way to control the tumour cell population is to give a high vaccine level at the beginning of the treatment and then reduce the treatment after a specific period of time, determined as part of the solution of the optimal control problem. We also found that increasing the strength of the DC vaccine causes an increase in the number of $\mathrm{NK}$ cells and $\mathrm{CD} 8^{+} \mathrm{T}$ cells, which in turn reduces the growth of the tumour. Increasing the strength of the DC vaccine also reduces the required duration of the treatment.

\section{References}

[1] Bunimovich-Mendrazitsky, S., Shochat, E. and Stone, L. Mathematical model of BCG immunotherapy in superficial bladder cancer. Bulletin of Mathematical Biology, 69:1847-1870, 2007. doi:10.1007/s11538-007-9195-Z C665

[2] Burden, T., Ernstberger, J. and Fister, K. R. Optimal control applied to immunotherapy. Discrete and Continuous dynamical Systems-Series B, 4(1):135-146, 2004. doi:10.3934/dcdsb.2004.4.135 C665, C666

[3] Cappuccio, A., Elishmereni, M. and Agur, Z. Cancer immunotherapy by interleukin-21: Potential treatment strategies evaluated in mathematical model. Cancer Res., 66(14):7293-7300, 2006. doi:10.1158/0008-5472.CAN-06-0241 C665

[4] Cappuccio, A., Castiglione, F. and Piccoli, B. Determination of the optimal therapeutic protocols in cancer immunotherapy. Mathematical Biosciences, 209(1):1-13, 2007. doi:10.1016/j.mbs.2007.02.009 C665 
[5] Castiglione, F. and Piccoli, B. Optimal control in a model of dendritic cell transfection cancer immunotherapy. Bulletin of Mathematical Biology, 68(2):255-274, 2006. doi:10.1007/s11538-005-9014-3 C665, C666, C667, C668

[6] de Pillis, L. G., Gu, W. and Radunskaya, A. E. Mixed immunotherapy and chemotherapy of tumours: modeling, applications and biological interpretations. Journal of Theoretical Biology, 238:841-862, 2006. doi:10.1016/j.jtbi.2005.06.037 C665, C668, C669

[7] de Pillis, L. G., Mallet, D. G. and Radunskaya, A. E. Spatial Tumor-Immune Modeling. Computational and Mathematical Methods in Medicine, 7(2-3):159-176, 2006. doi:10.1080/10273660600968978 C665

[8] de Pillis, L. G., Radunskaya, A. E. and Wiseman, C. L. A validated mathematical model of cell-mediated immune response to tumour growth. Cancer Research, 65:7950-7958, 2005.

http://www.ncbi.nlm.nih.gov/pubmed/16140967 C668

[9] El-Gohary, A. Chaos and optimal control of equilibrium states of tumor system with drug, Chaos, Soliton and Fractals, 41:425-435, 2009. doi:10.1016/j.chaos.2008.02.003 C665, C666

[10] Ghaffari, A. and Naserifar, N. Mathematical modeling and Lyapunov-based drug administration in cancer chemotherapy. Iranian Journal of Electrical and Electronic Engineering, 5(3):151-158, 2009. http://www.sid.ir/en/VEWSSID/J_pdf/106520090310.pdf C665, C666

[11] Ghaffari, A. and Naserifar, N. Optimal therapeutic protocols in cancer immunotherapy. Computers in Biology and Medicine, 40:261-270, 2010. doi:10.1016/j.compbiomed.2009.12.001 C665, C666

[12] Isaeva, O. G. and Osipov, V. A. Modelling of anti-tumour immune response: Immunocorrective effect of weak centimetre electromagnetic 
waves. Computational and Mathematical Methods in Medicine, 10:185-201, 2009. doi:10.1080/17486700802373540 C665

[13] Kirschner, D. and Panetta, J. C. Modeling immunotherapy of the tumor-immune interaction. J. Math. Biol., 37:235-252, 1998. doi:10.1007/s002850050127 C665

[14] Kuznetsov, V. A., Makalkin, I. A., Taylor, M. A. and Perelson, A. S. Nonlinear dynamics of immunogenic tumors: Parameter estimation and global bifurcation analysis. Bulletin of Mathematical Biology, 56:295-321, 1994. doi:10.1007/BF02460644 C668

[15] N. Larmonier, J. Fraszack, D. Lakomy, Bonnotte, B. and Katsanis, E. Killer dendritic cells and their potential for cancer immunotherapy. Cancer Immunology Immunotherapy, 59:1-11, 2010. doi:10.1007/s00262-009-0736-1 C667

[16] Lenhart, S. and Workman, J. T. Optimal control applied to biological models. Chapman \& Hall/CRC Mathematical and Computational Biology Series, 2007.

http://www.crcpress.com/product/isbn/9781584886402 C670

[17] Mallet, D. G. and de Pillis, L. G. A cellular automata model of tumour-immune system interactions. Journal of Theoretical Biology, 239:334-350, 2006. doi:10.1016/j.jtbi.2005.08.002 C665

[18] Moretta, A. Natural killers and dendritic cells: rendezvous in abused tissues. Nat. Rev. Immunol., 2(12):957-964, 2002. doi:10.1038/nri956 C667

[19] Murray, J. M. Some optimal control problems in cancer chemotherapy with a toxicity limit. Mathematical Biosciences, 100:49-67, 1990. doi:10.1016/0025-5564(90)90047-3 C666

[20] Swan, G. W. Role of optimal control theory in cancer chemotherapy. Mathematical Biosciences, 101:237-284, 1990. doi:10.1016/0025-5564(90)90021-P C666 
[21] Swierniak, A., Ledzewicz, U. and Schättler, H. Optimal control for a class of compartmental models in cancer chemotherapy. Int. J. Appl. Math. Comput. Sci., 13(3):357-368, 2003. https://www . amcs . uz .zgora.pl/?action=paper\&paper=154 C666

[22] Wu, Y., Xia, L., Zhang, M. and Zhao, X. Immunodominance analysis through interactions of $\mathrm{CD}^{+} \mathrm{T}$ cells and DCs in lymph nodes. Math. Biosci., 225(1):53-38, 2010. doi:10.1016/j.mbs.2010.01.009 C667, C668

\section{Author addresses}

1. Trisilowati, Mathematical Sciences School, Queensland University of Technology, Queensland 4000, Australia, and Department of Mathematics, Brawijaya University, Jalan Veteran Malang 65145, Indonesia.

2. Scott W. McCue, Mathematical Sciences School, Queensland University of Technology, Queensland 4000, Australia.

3. Dann G. Mallet, Mathematical Sciences School, Queensland University of Technology, Queensland 4000, Australia. mailto:dg.mallet@qut.edu.au 